\title{
A Soft X-ray Spectrometer using a Highly Dispersive Multilayer Grating
}

\author{
Tony W arwick, Howard Padmore, D mitriy V oronov and V aleriy Y ashchuk
}

Advanced Light Source, Lawrence Berkeley National Laboratory, Berkeley, CA94720, USA

\begin{abstract}
There is a need for higher resolution spectrometers as a tool for inelastic x-ray scattering. Currently, resolving power around $R=10,000$ is advertised. M easured $R I X S$ spectra are often limited by this instrumental resolution and higher resolution spectrometers using conventional gratings would be prohibitively large. We are engaged in a development program to build blazed multilayer grating structures for diffracting soft x-rays in high order. This leads to spectrometers with dispersion much higher than is possible using metal coated-gratings. The higher dispersion then provides higher resolution and the multilayer gratings are capable of operating away from grazing incidence as required. A spectrometer design is presented with a total length $3.8 \mathrm{~m}$ and capable of $10^{5}$ resolving power.
\end{abstract}

K eywords: RIXS inelastic $X$-ray scattering spectrometer multilayer gratings

PACS: 07.85.Fv, 07.85.Qe

\section{BLAZED MULTILAYER GRATINGS IN HIGH DIFFRACTED ORDER}

Gratings [1] will consist of a blazed substrate with a multilayer coating. The blaze angle and multilayer period is chosen so that the appropriate diffracted order at the required photon energy is on-blaze and at the same time satisfies the Bragg condition for efficient reflection from the tilted multilayer surface. The spectral bandwidth for efficient multilayer reflection is the Bragg bandwidth. This is about equal to the inverse of the number of layers participating. Typically 50 layers participate at soft x-ray energies [2]. This is a good match to the spectrometer bandwidth required for a typical RIXS experiment. The emission energy is fixed by the resonant condition from a particular atomic species (eg $\mathrm{Cu}, \mathrm{Mn}, \mathrm{Fe}, \mathrm{O}$ ). Operation is in high diffracted order $(\mathrm{m})$ to achieve the dispersion required, depending on the period of the underlying blazed structure. The effective grating period is $\mathrm{d}_{g} / \mathrm{m}$. The spectrometer study below uses $d_{g} / m=30 n m$. These gratings offer the potential for high dispersion and high resolution (see below). The downside is that a specific grating is needed for RIXS from a particular atomic species.

We have employed the code GSO LVER [3] to compute the diffraction efficiency using electromagnetic theory with a meshed model of the groove geometry. This model can include the trailing edge shape and includes $x$-ray penetration into the bulk for a more accurate assessment of the shadowing effects. The top surface of these gratings could be polished flat, eliminating shadowing. Our GSOLVER modeling reveals the extent of the benefit of this. The most important energy range for RIX S is between $250 \mathrm{eV}$ and $1 \mathrm{keV}$. As the photon energy increases the multilayer period is reduced and operation is closer to grazing, to satisfy the $B$ ragg condition $n \lambda=2 d_{m} \cos \theta(n=1)$.



FIGURE 1. Geometry of a single grating period. Sign convention: $\beta<0$ and $\mathrm{m}>0$. 
At $1 \mathrm{keV}$, operation is close to the limit imposed by the smallest practical multilayer period (say $d_{m}>3 n m$ for good efficiency) and close to the limit of the horizon at $\alpha=90^{\circ}$ (say $\propto<87.5^{\circ}$ for reasonable angular acceptance). As a stringent test, this study is made at high energy $(940 \mathrm{eV}, \mathrm{Cu}$ L-edge RIX S) where the (W/Si) multilayer is most difficult and the operational parameters of the spectrometer most restricted (see below).

Figure 2 shows the computed efficiency of the high dispersion grating in the spectrometer to be studied. $d_{g}=$ $150 \mathrm{~nm}, m=5, d_{g} / m=30 \mathrm{~nm}$. $\theta$ is half the included angle at the grating (see Figure 1). At $940 \mathrm{eV}$ there is little choice for $\theta$ if the multilayer period is to be larger than $3 \mathrm{~nm}$ and operation is to be above the horizon, which is at $\theta=81.5^{\circ}$. The operational range for $\theta$ shrinks to zero at values of $d_{g} / \mathrm{m}$ smaller than about $30 \mathrm{~nm}$. This sets a limit to the maximum dispersion at $940 \mathrm{eV}$. This limit is eased at lower photon energy.

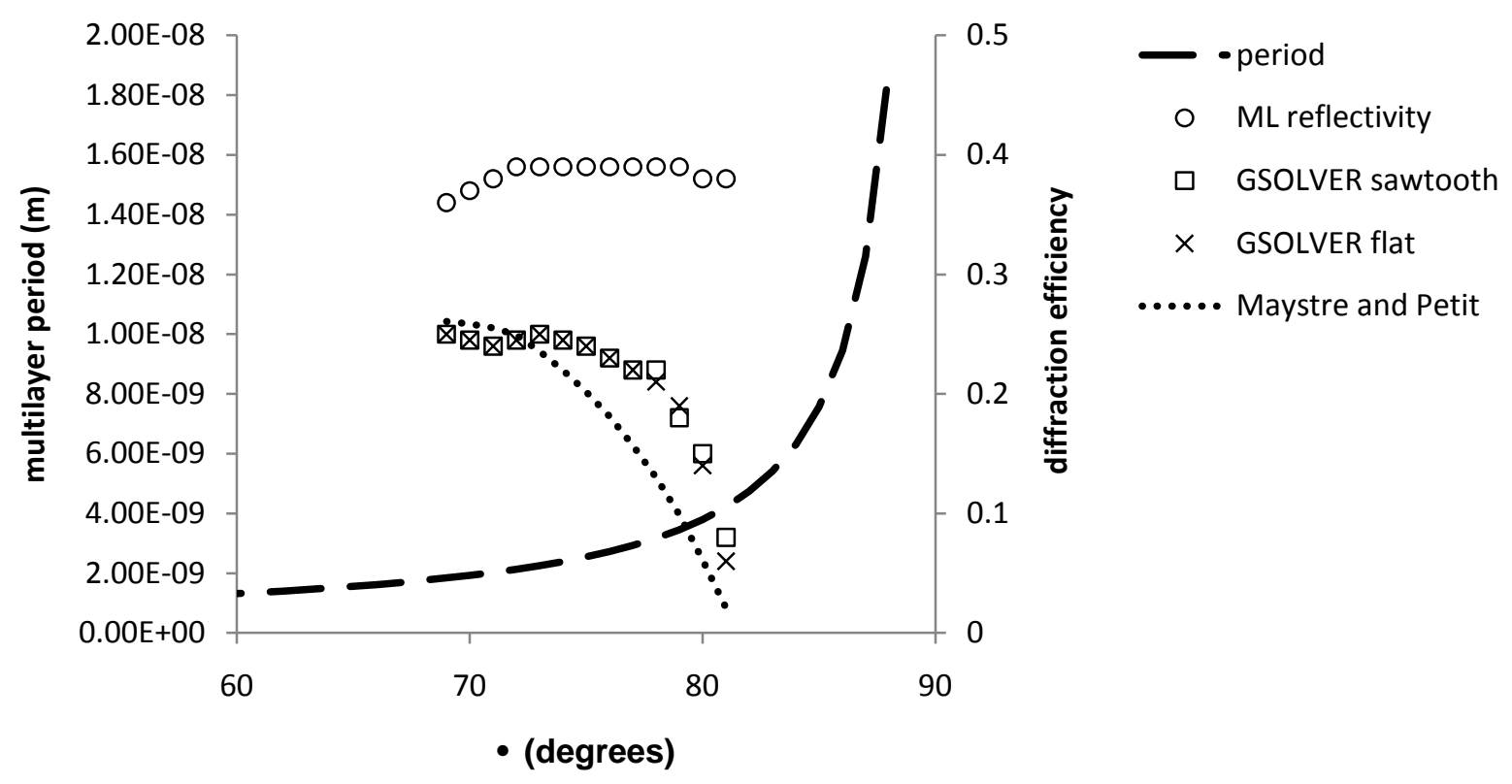

FIG URE 2. Multilayer period, computed reflectivity and diffraction efficiency for a blazed W/Si multilayer grating on-blaze at $940 \mathrm{eV}$ with $\mathrm{d}_{\mathrm{o}} / \mathrm{m}=30 \mathrm{~nm}$. The operational range of theta is restricted to around $80^{\circ}$, above the horizon at $81.5^{\circ}$ and so that the multilayer period is $>3 \mathrm{~nm}$. The computation of multilayer period ignores refraction, but this is included in the GSOLVER efficiency computations. There is no significant difference between flat and sawtooth.

\section{SPE CTROMETER DESIGN}

This is a futuristic design so we make some optimistic assumptions about parameter values and about grating availability. We use a varied line space (VLS) to correct the aberrations of a spherical multilayer grating. Unlike in a Rowland circle mount, we can select the arm length ratio to fit the slit size and detector resolution and we can select the angle of the focal plane. This seems the best spectrometer choice and is the same scheme adopted at SLS [4].

The spot size on the sample is $5 \mu \mathrm{m}$ (or use $5 \mu \mathrm{m}$ spectrometer entrance slits). We anticipate a high resolution imaging detector laying along the spectrometer focal plane inclined at $30^{\circ}$ to the optical axis giving $3 \mu \mathrm{m}$ transverse resolution and the promise of reasonable detector efficiency because the photons can penetrate any dead layer. The range of photons in Si at $940 \mathrm{eV}$ is $2.5 \mu \mathrm{m}$ which represents $0.4 \mu \mathrm{m}$ smearing due to the $30^{\circ}$ detector tilt.

The design is for $940 \mathrm{eV}(\mathrm{Cu})$ as the highest, most challenging, photon energy. From the discussion of the grating properties, we take $d_{g} / \mathrm{m}=30 \mathrm{~nm}$ and use $\theta=80^{\circ}$. We imagine a $150 \mathrm{~nm}$-period grating $50 \mathrm{~mm}$ long in 5th diffracted order. A coarser grating in higher order would be optically equivalent. With this dispersion, we size the spectrometer $(3.8 \mathrm{~m}$ long) so that the 5 micron entrance slit and the 3 micron detector resolution are equivalent and the so that the slit/detector limited resolving power is $10^{5}$. 
W e choose the V LS linear variation and the grating radius together to bring the spectrometer into focus at $940 \mathrm{eV}$ and tilt the focal plane by $30^{\circ}$. We choose the quadratic VLS variation to minimize the aberrations, principally coma. We find a cubic term unnecessary.

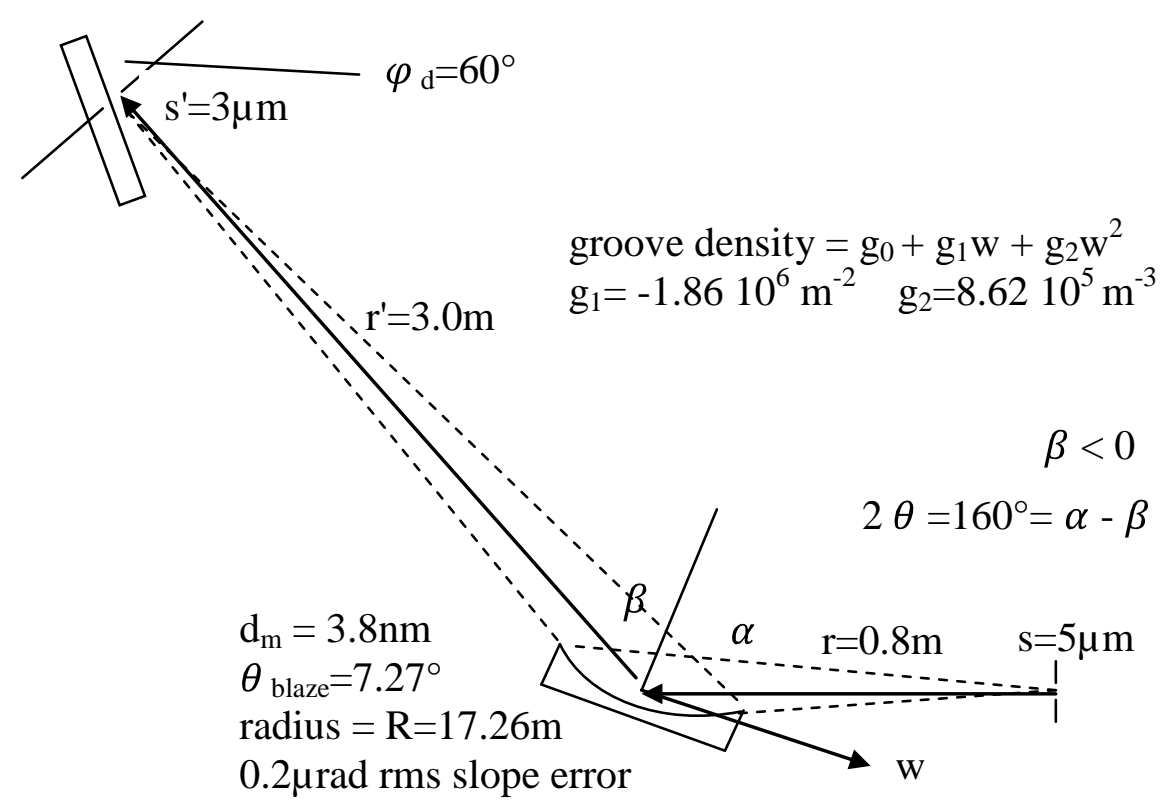

FIGURE 5. G eometry of the $3.8 \mathrm{~m}$ multilayer spectrometer and definition of variables.

Focusing and aberration canceling design of the V LS follows references [5], [6]. The important terms in the optical path function for the spherical grating are:

$$
\begin{gathered}
\mathrm{F}=\mathrm{r}+\mathrm{r}^{\prime}+\mathrm{nm} \lambda-\mathrm{w} \sin \alpha-\mathrm{w} \sin \beta+\frac{\mathrm{w}^{2}}{2}\left[\mathrm{~T}_{\alpha}+\mathrm{T}_{\beta}\right]+\frac{\mathrm{w}^{3}}{2}\left[\frac{\mathrm{T}_{\alpha} \sin \alpha}{\mathrm{r}}+\frac{\mathrm{T}_{\beta} \sin \beta}{\mathrm{r}^{\prime}}\right]+\ldots \ldots \ldots \ldots . . . . . . \\
\mathrm{T}_{\alpha}=\frac{\cos ^{2} \alpha}{\mathrm{r}}-\frac{\cos \alpha}{\mathrm{R}}
\end{gathered}
$$

$n$ counts grooves from the center of the grating in the direction of $w$. The groove density is:

$$
\frac{d n}{d w}=g_{0}+g_{1} w+g_{2} w^{2}+g_{3} w^{3}+\ldots \ldots \ldots
$$

Setting the derivative with respect to $w$ of the optical path to zero and equating coefficients yields:

$$
\begin{gathered}
\mathrm{m} \lambda \mathrm{g}_{0}=\sin \alpha+\sin \beta \quad \text { grating equation } \\
\frac{\cos ^{2} \alpha}{\mathrm{r}}+\frac{\cos ^{2} \beta}{\mathrm{r}^{\prime}}-\frac{\cos \alpha+\cos \beta}{\mathrm{R}}+\mathrm{m} \lambda \mathrm{g}_{1}=0 \text { focusing condition } \\
\frac{3}{2}\left[\frac{\mathrm{T}_{\alpha} \sin \alpha}{\mathrm{r}}+\frac{\mathrm{T}_{\beta} \sin \beta}{\mathrm{r}^{\prime}}\right]+\mathrm{g}_{2} \mathrm{~m} \lambda=0 \text { coma correction }
\end{gathered}
$$


The radius of the grating to achieve focus is then:

$$
\mathrm{R}=\frac{\frac{1}{\mathrm{~m} \lambda}[\cos \alpha+\cos \beta]+g_{0} \tan \beta}{\frac{1}{\mathrm{~m} \lambda}\left[\frac{\cos ^{2} \alpha}{r}+\frac{\cos ^{2} \beta}{r^{\prime}}\right]+\frac{2 g_{0} \sin \beta}{r^{\prime}}+\frac{g_{0} \cos \beta \tan \varphi_{\mathrm{d}}}{r^{\prime}}}
$$

And the slope of the focal plane is found by differentiating the focusing condition and the grating equation with respect to $\beta$ :

$$
\tan \varphi_{\mathrm{d}}=\frac{1}{\mathrm{r}^{\prime}} \frac{\partial \mathrm{r}^{\prime}}{\partial \beta}=\frac{\mathrm{r}^{\prime}}{\cos \beta}\left[\frac{\tan \beta}{\mathrm{R}}+\frac{\mathrm{g}_{1}}{\mathrm{~g}_{0}}-\frac{2 \sin \beta}{\mathrm{r}^{\prime}}\right]
$$

As reported in reference [4] we find, when raytracing, that the coma correcting value of $g_{2}$ (given in Figure 5) is not the best choice. Empirically determined values perform slightly better, presumably by balancing higher order aberrations. Raytracing using SHADOW [7] shows no benefit from including the cubic VLS term $g_{3}$. Figure 6. shows raytrace images on the face of the detector, verifying that the resolution is maintained along the inclined focal plane.
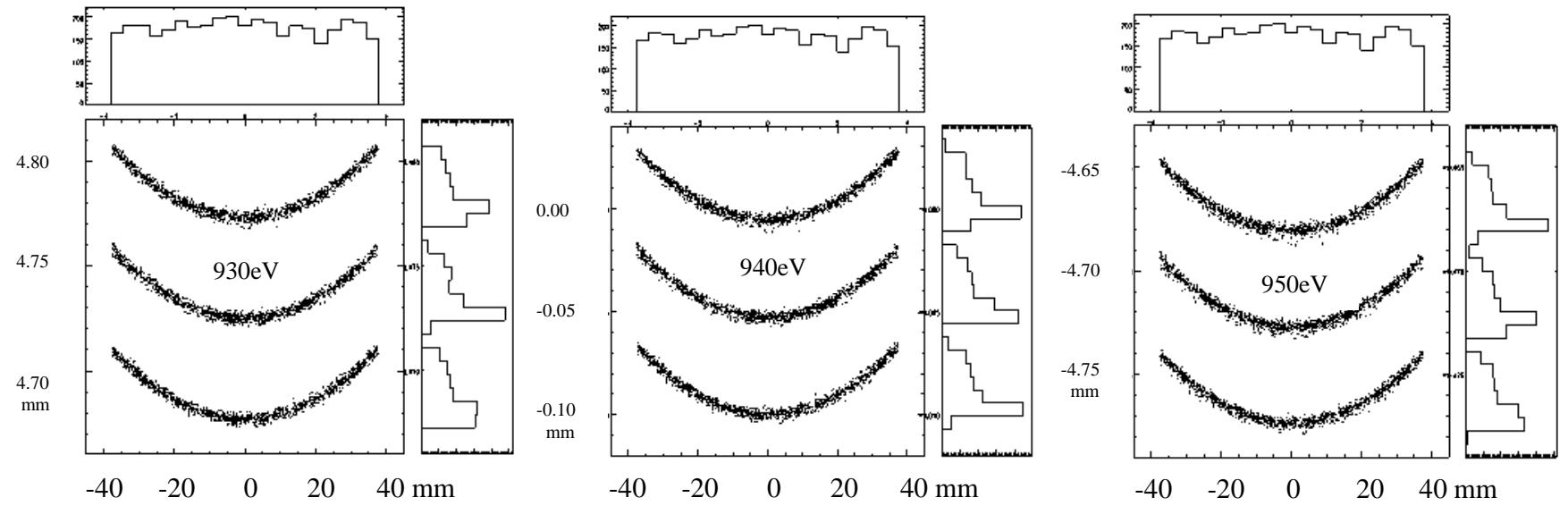

FIGURE 6. Raytracing using SHADOW [7] at $930 \mathrm{eV} \pm 100 \mathrm{meV}, 940 \mathrm{eV} \pm 100 \mathrm{meV}$ and $950 \mathrm{eV} \pm 100$ meV maintaining the resolution along a focal plane inclined at $30^{\circ}$ to the optical axis. The figure shows rays incident on three areas of the focal plane separated by about $5 \mathrm{~mm}$. Horizontal and vertical collection angles are $20 \mathrm{mrad}$ and $3 \mathrm{mrad}$ respectively. These images are $76 \mathrm{~mm}$ wide at the detector, which needs to extend $10 \mathrm{~mm}$ al ong the focal plane.

\section{CONCLUSIONS}

Higher resolution x-ray emission spectrometers can be designed using high dispersion gratings, without becoming larger. M ultilayer coated gratings are essential to maintain reasonable efficiency at the smaller values of the included angle that are required. There is a limit to the useful dispersion of the grating, arising from the smallest period multilayers available. This limit is more restrictive as the photon energy increases. Within this limit, spectrometers can be designed with resolution about ten times that currently available from those using metal gratings, without increasing their size. 
For an explicit comparison, a spherical VLS grating spectrometer was considered using a conventional Aucoated blazed grating with lower dispersion (3333 lines/mm in first order) to achieve the same resolving power $\left(10^{5}\right.$ ) with the same size slits $(5 \mu \mathrm{m}$ and $3 \mu \mathrm{m})$ at shallower angles $\left(\theta=87^{\circ}\right)$. This conventional instrument was $11.2 \mathrm{~m}$ long, and would require a $500 \mathrm{~mm}$ grating to achieve the same $3 \mathrm{mrad}$ angular acceptance as in the $3.8 \mathrm{~m}$ multilayer design. The rms slope error tolerance for the Au grating would be $0.07 \mu \mathrm{rad}$. The angular acceptance in the non dispersive direction is set by the width of the detector and is reduced by a factor three because of the increased spectrometer length. The gold coated grating is $4.2 \%$ efficient at $940 \mathrm{eV}$ in this grazing geometry, whereas we compute $14 \%$ efficiency at $940 \mathrm{eV}$ for the multilayer grating in the $3.8 \mathrm{~m}$ spectrometer.

\section{ACKNOWLEDGMENTS}

This work was supported by the U.S. Department of E nergy under contract number DE - A C 02-05CH 11231.

\section{REFERENCES}

1. D. L. Voronov, R. Cambie, R. M. Feshchenko, E. M. Gullikson, H. A. Padmore, A. V. V inogradov and V. Y. Y ashchuk Proc. of SPIE V ol. 6705 67050E

2. E.M. Gullikson, Center for X-ray Optics, Lawrence B erkeley Laboratory http://henke.Ibl.gov/optical_constants/

3. D Fluckiger - Grating Solver Development Company Dec 2006 http://www.gsolver.com

4. G. Ghiringhelli, A. Piazzalunga, C. Dallera, G. Trezzi, L. B raicovich, T. Schmitt, V.N. Strocov, R. B etemps, L. Patthey, X. W ang and M. Grioni, Phys. Rev. Lett. 102 (2009) 027401

5. K. A memiya, Y. K itajima, T. O hta and K. Ito, J. Synchrotron Radiation 3 (1996) 282

6. Y.-D. Chaung, J. Pepper, W. M ckinney, Z Hussain, E Gullikson, P. B atson, D, Qian and M. Z. Hasan J. Phys. Chem. Solids 66 (2005) 2173

7. The original SHADOW package is available at http://www.nanotech.wisc.edu/CNTLABS/shadow.html and with an IDL user interface at http://www.esrf.fr/computing/scientific/xop 